\title{
CUBIC MECHANICAL METHOD FOP THE NONLINEAR SYSTEM OF SINGULAR INTEGRAL EQUATIONS
}

\author{
EISSA R.P. AND GAD M.M.
}

Many applied problems in the theory of elasticity can be reduced to the solution of singular integral equations either linear or nonlinear.

In this paper we shall study a nonlinear system of singular integral equations which appear on the closed Lipanouv surface in an ideal medium [4].

We shall find a cubic mechanical method which corresponds to the system and prove its convergence; we obtained a discrete operator which corresponds to this system and study its properties and then a solution to the resulting system of the nonlinear equations which leads to an approximate solution for the original system and its convergence.

\section{Introduction}

Consider the two dimensional nonlinear singular integral equations system

$$
\left\{\begin{array}{l}
U(P)-\lambda A U-\lambda B V=f(P), \\
V(P)-\lambda C U-\lambda D V=g(P)
\end{array}\right.
$$

where $A, B, C$ and $D$ are nonlinear singular operators of the type

$$
\int_{S} \frac{K(P, Q)}{r^{2}(P, Q)} \Phi(U(Q)) d S_{Q}
$$

where $S \in \ell_{1}(\delta)$ be the closed Lipanouv surface in $R^{3}$,

$$
\delta \in(0,1] ; P\left(x_{1}, x_{2}, x_{3}\right), Q\left(y_{1}, y_{2}, y_{3}\right) \in S, r(P, Q)=\left[\sum_{i=1}^{3}\left(x_{i}-y_{i}\right)^{2}\right]^{\frac{1}{2}}
$$

Let $L_{i}(i=0,1,2)=$ constants $>0$, such that for all $P, P_{1}, P_{2}, Q, Q_{1}$ and $Q_{2} \in S$ the following relations are satisfied:

(a) $|K(P, Q)| \leq \bar{\nu}_{0}$;

Received December 5, 1989. 
(b) $\left|K\left(P_{1}, Q\right)-K\left(P_{2}, Q\right)\right| \leq L_{1} r\left(P_{1}, P_{2}\right) \rho_{Q}^{-1}\left(P_{1}, P_{2}\right)$;

(c) $\left|K\left(P, Q_{1}\right)-K\left(P, Q_{2}\right)\right| \leq L_{2} r^{\delta}\left(Q_{1}, Q_{2}\right) \rho_{P}^{-\delta}\left(Q_{1}, Q_{2}\right)$;

where $\rho_{Q}\left(P_{1}, P_{2}\right)=\max \left\{r\left(P_{1}, Q\right), r\left(P_{2}, Q\right)\right\}, \rho_{P}\left(Q_{1}, Q_{2}\right)=\max \left\{r\left(P, Q_{1}\right), r\left(P, Q_{2}\right)\right\}$, $\Phi(t)$ is any function defined on the open interval $(-a, a)$ with the following conditions:

(1) $\Phi(0)=0$;

(2) the derivative $\frac{d \Phi}{d t}$ exists, and is bounded on $(-a, a)$;

(3) $\left|\Phi^{\prime}\left(t_{1}\right)-\Phi^{\prime}\left(t_{2}\right)\right| \leq L_{3}\left|t_{1}-t_{2}\right|$;

$\lambda$ is a numerical parameter, $U(P)$ and $V(P)$ are unknown functions from the Hölder class $H_{\alpha}(S)$; where

$$
\begin{aligned}
& H_{\alpha}(S)=\{Z(P) \in C(S): H(Z, \alpha)<\infty, \alpha \in(0,1)\}, \\
& H(Z, \alpha)=\max _{Q_{1}, Q_{2} \in S}\left|Z\left(Q_{1}\right)-Z\left(Q_{2}\right)\right| r^{-\alpha}\left(Q_{1}, Q_{2}\right) ;
\end{aligned}
$$

$f(P)$ and $g(P) \in H_{\alpha}(S)$ are given functions.

$H_{\alpha}(S)$ is a B-space with the norm

$$
\|Z\|_{\alpha}=\|Z\|_{c}+H(Z ; \alpha) .
$$

(In what follows we consider $\alpha<\delta$.)

In the integral

$$
W(U ; P)=\int_{S} \frac{K(P, Q)}{r^{2}(P, Q)} U(Q) d S_{Q},
$$

We take the principal value (P.V.P).

We shall use the characterstic function $f(P, \theta)$ which is obtained in [1], and is defined by: $f(P, \theta)=\lim _{r \rightarrow 0} K(P, r, \theta)$, where $\theta$ is the angle between the plane passing through the interval $r$ and the normal to the surface $S$ at the point $P$ and another fixed plane passing through the same normal; as in $[1,3]$, the singular integral (2) can be written in the form

$$
\begin{aligned}
W(U ; P) & =W_{1}(U ; P)+W_{2}(U, P), \text { where } \\
W_{1}(U, P) & =\int_{S} \frac{K(P, Q)-f(P, \theta)}{r^{2}(P, Q)} U(Q) d S_{Q}, \\
W_{2}(U ; P) & =\int_{S} \frac{f(P, \theta)}{r^{2}(P, Q)}(U(Q)-U(P)) d S_{Q} .
\end{aligned}
$$

It is clear that the first integral $W_{1}$ has a weak singularity, and the second $W_{2}$ is singular Define, the functions $M(P, Q)$ and $f(P, \theta)$ such that $M(P, Q) \stackrel{\text { def }}{=} K(P, Q)-f(P, \theta)$, where $M(P, Q)$ and $f(P, \theta)$ satisfies the following conditions; there exists $L_{1}(i=\overline{4,9})=$ constants $>0$, for all $P, P_{1}, P_{2}, Q, Q_{1}$ and $Q_{2} \in S$,

(i) $|M(P, Q)| \leq L_{4} r^{\delta}(P . Q)$; 
(ii) $\left|M\left(P_{1}, Q_{1}\right)-M\left(P_{2}, Q_{2}\right)\right| \leq L_{5} r^{\delta}\left(P_{1}, P_{2}\right)+L_{6} r^{\delta}\left(Q_{1}, Q_{2}\right)$;

(iii) $|f(P, \theta)| \leq L_{7}$;

(iv) $\left|f\left(P_{1}, \theta\right)-f\left(P_{2}, \theta\right)\right| \leq L_{8} r\left(P_{1}, P_{2}\right) \rho_{Q}^{-1}\left(P_{1}, P_{2}\right)$;

(v) $\left|f\left(P, \theta_{1}\right)-f\left(P, \theta_{2}\right)\right| \leq L_{9} r^{\delta}\left(Q_{1}, Q_{2}\right) \rho_{P}^{-\delta}\left(Q_{1}, Q_{2}\right)$.

where $\theta, \theta_{1}$ and $\theta_{2}$ corresponding to $Q, Q_{1}$ and $Q_{2}$ respectively

\section{The cubic formula for the singular integral (2) and its convergence.}

To find the cubic formula and prove its convergency we use the results obtained in $[5]:$

Pratitioning $S$ : Let the equation of the closed Lipanouv surface $S \in \ell_{1}(\delta)$ in the parametric form as follows:

$x_{i}=x_{i}(U, V), i=1,2,3$; where $x_{i}(U, V)$ are continuous and differentiable functions defined on $\Delta$, where $\Delta$ is the closed range of the variables $U$ and $V$.

In the same way $S$ can be divided by the linear coordinates $U(n)$ and $V(n)$ into subdomains $S\left(P_{k}\right), k=1,2, \cdots, N_{n}$; with support points $P_{k}$ where $n$ is the parameter of division, $n \geq n_{0}$. Moreover we defined the following:

for all $k, k_{1}, k_{2} \in\left\{1,2, \cdots, N_{n}\right\}$,

$$
r_{k}(n)=R_{k}(n), \quad r_{k_{1}}(n)=r_{k_{2}}(n),
$$

where

$$
\begin{aligned}
r_{K}(n) & =\inf _{Q \in \partial S\left(P_{K}\right)}\left\{r\left(P_{K}, Q\right)\right\} ; \\
R_{K}(n) & =\sup _{Q \in S\left(P_{K^{\prime}}\right)}\left\{r\left(P_{K}, Q\right)\right\} ; \\
\lim _{n \rightarrow \infty} r_{k}(n) & =\lim _{n \rightarrow \infty} R_{K}(n)=0 ;
\end{aligned}
$$

we choose $n_{0}$ such that for all $k, R_{K}(n)<d$, where $d$ is the standard radius of Lipanouv sphere.

$a_{n}=b_{n}$ means that there exists $\ell_{1}, \ell_{2}=$ constants $>0$, such that for all $n>n_{n}$.

$\ell_{1} a_{n} \leq b_{n} \leq \ell_{2} a_{n}$.

Now we construct the cubic formula for the equation (2).

Let

$$
\begin{aligned}
& W_{1}^{(n)}\left(U ; P_{\ell}\right) \stackrel{\text { def }}{=} \sum_{k=1, k \neq \ell}^{N_{n}} \frac{K\left(P_{\ell}, P_{K}\right)-f\left(P_{\ell}, \theta P_{K}\right)}{r^{2}\left(P_{\ell}, P_{K}\right)} U\left(P_{K}\right) \text { mes } S\left(P_{K}\right) ; \\
& W_{2}^{(n)}\left(U ; P_{\ell}\right) \stackrel{\text { def }}{=} \sum_{k=1, k \neq \ell}^{N_{n}} \frac{f\left(P_{\ell}, \theta P_{K}\right)}{r^{2}\left(P_{\ell}, P_{K}\right)}\left(U\left(P_{K}\right)-U\left(P_{\ell}\right)\right) \text { mes } S\left(P_{K}\right) ;
\end{aligned}
$$

We take

$$
W^{(n)}\left(U ; P_{\ell}\right)=W_{1}^{(n)}\left(U ; P_{\ell}\right)+W_{2}^{(n)}\left(U ; P_{\ell}\right)
$$




$$
\begin{aligned}
= & \sum_{k=1, k \neq \ell}^{N_{n}} \frac{K\left(P_{\ell}, P_{K}\right)}{r^{2}\left(P_{\ell}, P_{K}\right)} U\left(P_{K}\right) \text { mes } S\left(P_{K}\right) \\
& -U\left(P_{\ell}\right) \sum_{k=1, k \neq \ell}^{N_{n}} \frac{f\left(P_{\ell}, \theta P_{K}\right)}{r^{2}\left(P_{\ell}, P_{K}\right)} \text { mes } S\left(P_{K}\right) ;
\end{aligned}
$$

as the cubic formula for equation (2) at the support points $P_{\ell}, \ell=1,2, \cdots, N_{n}$. Now we extend this formula at all points of the surface $S$, setting

$$
W^{(n)}(U ; P)=W^{(n)}\left(U ; P_{\ell}\right) \text {, if } P \in S\left(P_{\ell}\right) \text {. }
$$

So, the remainder value of the formula (5),(6):

$$
\begin{aligned}
R^{(n)}(U ; P) & \stackrel{\text { def }}{=} W(U ; P)-W^{(n)}(U ; P) \\
& =R_{1}^{(n)}(U ; P)+R_{2}^{(n)}(U ; P) \\
& =\left(W_{1}(U ; P)-W_{1}^{(n)}(U ; P)\right)+\left(W_{2}(U ; P)-W_{2}^{(n)}(U ; P)\right)
\end{aligned}
$$

Let

$$
R(n)=\max _{k=1, N_{n}}\left\{R_{K}(n)\right\}, r(n)=\min _{k=1, N_{n}}\left\{r_{k}(n)\right\} .
$$

We obtain the following theorems which are analogs to the results in [5].

Theorem 1. For all $n \geq n_{0}$, we have:

$$
\underline{\max }_{\ell=l, N_{n}}\left|R^{(n)}\left(U ; P_{\ell}\right)\right| \leq C_{0}\|U\|_{\alpha} R^{\alpha}(n) \operatorname{Ln} \frac{l}{r(n)}
$$

Theorem 2. For all $n \geq n_{0}$, we have:

$$
\operatorname{Sup}_{P \in S}\left|R^{(n)}(U ; P)\right| \leq C_{l}\|U\|_{\alpha} R^{\alpha}(n) \operatorname{Ln} \frac{l}{r(n)}
$$

where in general $C_{i}, i=0,1,2, \cdots$, are constants independently of $n$.

3. The discrete operator and its properties.

Let $R_{N_{n}}$ - be a vector space with dimension $N_{n}$ whose elements are of the form

$$
U_{N_{n}}=\left(U\left(P_{1}\right), U\left(P_{2}\right), \ldots, U\left(P_{N_{n}}\right)\right)
$$


We define the nommin this space as follows

$$
\begin{aligned}
\left\|U_{N_{n}}\right\|_{\alpha}^{\left(N_{n}\right)} & =\max _{i=1, N_{n}}\left|U\left(P_{i}\right)\right|+H^{\left(N_{n}\right)}\left(U_{N_{n}} ; \alpha\right), \text { where } \\
H^{\left(N_{n}\right)}\left(U_{N_{n}} ; \alpha\right) & =\sup _{\rho>0} \rho^{-\alpha} \bar{\omega}^{\left(N_{n}\right)}\left(U_{N_{n}} ; \rho\right), \\
\bar{\omega}^{\left(N_{n}\right)}\left(U_{N_{n}} ; \rho\right) & =\sup _{\xi \geq \rho} \xi^{-1} \omega^{\left(N_{n}\right)}\left(U_{N_{n}}, \xi\right)
\end{aligned}
$$

is a majorant for the continuity modlus of the vector $U_{N_{n}}$,

$$
\omega^{\left(N_{n}\right)}\left(U_{N_{n}} ; \rho\right)=\max _{r\left(P_{k}, P_{\ell}\right)<\rho, k, \ell=\overline{1, N_{n}}}\left|U\left(P_{K}\right)-U(P)\right|, 0<\rho<\operatorname{diam} S
$$

and $\omega^{\left(N_{n}\right)}\left(U_{N_{n}} ; \rho\right)=0$ if $\rho<\min _{k \neq \ell}\left\{r\left(P_{K}, P_{\ell}\right)\right\}$.

It is clear that:

$$
\left(R_{N_{n}},\|\cdot\|_{\alpha}^{\left(N_{n}\right)}\right) \equiv H_{\alpha}^{\left(N_{n}\right)} \text { is a B-space. }
$$

consider now the vector

$$
W^{(n)} U_{N_{n}}=\left(W^{(n)}\left(U_{N_{n}} ; P_{1}\right), W^{(n)}\left(U_{N_{n}} ; P_{2}\right), \ldots, W^{(n)}\left(U_{N_{n}} ; P_{N_{n}}\right)\right)
$$

with

$$
W^{(n)}\left(U_{N_{n}} ; P_{i}\right)=W^{(n)}\left(U ; P_{i}\right), \quad i=\overline{1, N_{n}}
$$

Equation (8) can be writen by using (3) and (4) as:

$$
\begin{aligned}
W_{1}^{(n)} U_{N_{n}} & \left.=\left(W_{1}^{(n)}\left(U_{N_{n}}, P_{1}\right), W_{1}^{(n)}\left(U_{N_{n}}\right), P_{2}\right), \cdots, W_{1}^{(n)}\left(U_{N_{n}} ; P_{N_{n}}\right)\right) ; \\
W_{2}^{(n)} U_{N_{n}} & \left.=\left(W_{2}^{(n)}\left(U_{N_{n}} ; P_{1}\right), W_{2}^{(n)}\left(U_{N_{n}}\right) ; P_{2}\right), \cdots, W_{2}^{(n)}\left(U_{N_{n}} ; P_{N_{n}}\right)\right) ; \\
W_{1}^{(n)}\left(U_{N_{n}} ; P_{i}\right) & =W_{1}^{(n)}\left(U ; P_{i}\right) \text { and } W_{2}^{(n)}\left(U_{N_{n}} ; P_{i}\right)=w_{2}^{(n)}\left(U ; P_{i}\right), i=\overline{1, N_{n}},
\end{aligned}
$$

Definition 1. The correspondence $W_{1}^{(n)}$ of every vector $U_{N_{n}}$ in the form (7) with the vector in the form (9) is called a discrete operator with weak singularity.

Definition 2. The correspondence $W_{2}^{(n)}$ of every vector $U_{N_{n}}$ in the form (7) with the vector in the form (10) is called a discrete singular operator.

As [1] we can obtain the following Lemma.

\section{Lemma 1.}

$$
\left\|W_{l}^{(n)} U_{N_{n}}\right\|_{\alpha}^{\left(N_{n}\right)} \leq C_{2}\left\|U_{N_{n}}\right\|_{\alpha}^{\left(N_{n}\right)}
$$

the proof is given if we put $h_{0}^{\prime}=\frac{(1 / 2) \operatorname{diam} S}{2+1 / C_{0}^{\prime}}$ in lemma $\mathscr{Q}^{\prime}$ [5] instead of $h_{0}[1]$. 


\section{Lemma 2.}

$$
\left\|W_{2}^{(n)} U_{N_{n}}\right\|_{\alpha}^{\left(N_{n}\right)} \leq C_{3}\left\|U_{N_{n}}\right\|_{\alpha}^{\left(N_{n}\right)} .
$$

From Lemmas 1 and 2 we have the following theorem.

Theorem 3.

$$
\left\|W^{(n)} U_{N_{n}}\right\|_{\alpha}^{\left(N_{n}\right)} \leq C_{4}\left\|U_{N_{n}}\right\|_{\alpha}^{\left(N_{n}\right)}
$$

4. The solution of the nonlinear algebraic system of equations.

We will write two lemmas which were proved in [2].

Lemma 3[2]. Let $\left\|U_{N_{n}}\right\|_{\alpha}^{\left(N_{n}\right)}<a$. Then

$$
\left\|\phi\left(U_{N_{n}}\right)\right\|_{\alpha}^{\left(N_{n}\right)} \leq C_{5}\left\|U_{N_{n}}\right\|_{\alpha}^{\left(N_{n}\right)} .
$$

Lemma 4[2]. Let $\left\|U_{N_{n}}^{(l)}\right\|_{\alpha}^{\left(N_{n}\right)}<a$ and $\left\|U_{N_{n}}^{(2)}\right\|_{\alpha}^{\left(N_{n}\right)}<a$. then

$$
\left\|\phi\left(U_{N_{n}}^{(l)}\right)-\phi\left(U_{N_{n}}^{(2)}\right)\right\|_{\alpha}^{\left(N_{n}\right)} \leq C_{6}\left\|U_{N_{n}}^{(l)}-U_{N_{n}}^{(2)}\right\|_{\alpha}^{\left(N_{n}\right)} .
$$

By applying the cubic formulae (5) and (6) to the system of the integral equations (1), then they will be written as

$$
\begin{aligned}
& U(P)-\lambda W^{(n)}\left(\phi_{A}(U) ; P\right)-\lambda R^{(n)}\left(\phi_{A}(U) ; P\right) \\
& -\lambda W^{(n)}\left(\phi_{B}(V), P\right)-\lambda R^{(n)}\left(\phi_{B}(V), P\right)=f(P) ; \\
& V(P)-\lambda W^{(n)}\left(\phi_{C}(U) ; P\right)-\lambda R^{(n)}\left(\phi_{C}(U) ; P\right) \\
& -\lambda W^{(n)}\left(\phi_{D}(V), P\right)-\lambda R^{(n)}\left(\phi_{D}(V) ; P\right)=g(P)
\end{aligned}
$$

Setting $P=P_{i}, \overline{i=1, N_{n}}$ and neglecting the remainder terms, we get the following system of nonlinear algebraic equations:

$$
\left\{\begin{array}{l}
w\left(P_{i}\right)-\lambda W^{(n)}\left(\phi_{A}(w) ; P_{i}\right)-\lambda W^{(n)}\left(\phi_{B}(Z), P_{i}\right)=f\left(P_{i}\right) ; \\
Z\left(P_{i}\right)-\lambda W^{(n)}\left(\phi_{C}(w) ; P_{i}\right)-\lambda W^{(n)}\left(\phi_{D}(Z), P_{i}\right)=g\left(P_{i}\right) ;
\end{array}\right.
$$

with $w\left(P_{i}\right)$ and $Z\left(P_{i}\right), i=\overline{1, N_{n}}$ the approximated values of $U\left(P_{i}\right)$ and $V\left(P_{i}\right)$.

Now we write system (11) in the vector form as follows

$$
\left\{\begin{array}{l}
w_{N_{n}}=\lambda W^{(n)} \phi_{A}\left(w_{N_{n}}\right)+\lambda W^{(n)} \phi_{B}\left(Z_{N_{n}}\right)+f_{N_{n}}, \\
Z_{N_{n}}=\lambda W^{(n)} \phi_{C}\left(W_{N_{n}}\right)+\lambda W^{(n)} \phi_{D}\left(Z_{N_{n}}\right)+g_{N_{n}}
\end{array}\right.
$$


In turns we write the system (12) in the opperator form

$$
\bar{E}_{N_{n}}=\lambda U \bar{E}_{N_{n}}+b_{N_{n}}
$$

where

$$
\begin{aligned}
\bar{E}_{N_{n}} & =\left(w_{N_{n}}, Z_{N_{n}}\right), \quad \bar{b}_{N_{n}}=\left(f_{N_{n}}, g_{N_{n}}\right) \quad \text { and } \\
U \bar{E}_{N_{n}} & =\left[W^{(n)} \phi_{A}\left(w_{N_{n}}\right)+W^{(n)} \phi_{B}\left(Z_{N_{n}}\right) ; W^{(n)} \phi_{C}\left(w_{N_{n}}\right)+W^{(n)} \phi_{D}\left(Z_{N_{n}}\right)\right] .
\end{aligned}
$$

Theorem 4. If $|\lambda|<\min \left\{\lambda_{1}, \lambda_{2}\right\}$, then the system of algebraic equations (13) has a unique solution. This solution can be found by using the method of iterated approximation. (The values of $\lambda_{1} \& \lambda_{2}$ are given below).

Proof. Let $\bar{E}_{N_{n}} \in B^{\left(N_{n}\right)}\left(\bar{b}_{N_{n}} ; a_{1}\right)$ be a sphere in $H_{\alpha}^{\left(N_{n}\right)} \times H_{\alpha}^{\left(N_{n}\right)}$ with radius $a_{1}$ and center $\bar{b}_{N_{n}}$.

Then we have

$$
\begin{aligned}
& \left\|\lambda U \bar{E}_{N_{n}}\right\| \stackrel{\text { def }}{=} \max \left\{\lambda\left\|W^{(n)} \phi_{A}\left(w_{N_{n}}\right)+\lambda W^{(n)} \phi_{B}\left(Z_{N_{n}}\right)\right\|_{\alpha}^{\left(N_{n}\right)} ;\right. \\
& \left.;\left\|\lambda W^{(n)} \phi_{C}\left(w_{N_{n}}\right)+\lambda W^{(n)} \phi_{D}\left(Z_{N_{n}}\right)\right\|_{\alpha}^{\left(N_{n}\right)}\right\} \leq \\
& <|\lambda| \max \left\{C_{4} C_{5}(A)\left(a_{1}+\left\|f_{N_{n}}\right\|_{\alpha}^{\left(N_{n}\right)}\right)+C_{4} C_{5}(B)\left(a_{1}+\left\|g_{N_{n}}\right\|_{\alpha}^{\left.N_{n}\right)}\right)\right. \\
& \left.; C_{4} C_{5}(C)\left(a_{1}+\left\|f_{N_{n}}\right\|_{\alpha}^{\left(N_{n}\right)}\right)+C_{4} C_{5}(D)\left(a_{1}+\left\|g_{N_{n}}\right\|_{\alpha}^{\left(N_{n}\right)}\right)\right\}<a_{1},
\end{aligned}
$$

for

$$
\begin{aligned}
& |\lambda| \leq a_{1} / C_{4}\left[\max \left\{C_{5}(A), C_{5}(C)\right\}\left(a_{1}+\| f_{N_{n}}\right) \|_{\alpha}^{\left(N_{n}\right)}\right)+ \\
& \left.+\max \left\{C_{5}(B), C_{5}(D)\right\}\left(a_{1}+\left\|g_{N_{n}}\right\|_{\alpha}^{\left(N_{n}\right)}\right)\right] \equiv \lambda_{1} ; \\
& \quad \text { i.e. }\left(\lambda U \bar{E}_{N_{n}}+\bar{b}_{N_{n}}\right) \in B^{\left(N_{n}\right)}\left(\bar{b}_{N_{n}} ; a_{1}\right) .
\end{aligned}
$$

Now we choose $\lambda$ such that the operator $\lambda U \bar{E}_{N_{n}}+\bar{b}_{N_{n}}$ will be a contraction operator;

for all $\bar{E}_{N_{n}}^{(1)}$ and $\bar{E}_{N_{n}}^{(2)} \in B^{\left(N_{n}\right)}\left(\bar{b}_{N_{n}}, a_{1}\right)$.

$$
\begin{aligned}
& \left\|\lambda U \bar{E}_{N_{n}}^{(1)}-\lambda U \bar{E}_{N_{n}}^{(2)}\right\| \stackrel{\text { def }}{=} \max \left\{\| \lambda W^{(n)} \phi_{A}\left(w_{N_{n}}^{(1)}\right)+\lambda W^{(n)} \phi_{B}\left(Z_{N_{n}}^{(1)}\right)-\right. \\
& \lambda W^{(n)} \phi_{A}\left(w_{N_{n}}^{(2)}\right)-\lambda W^{(n)} \phi_{B}\left(Z_{N_{n}}^{(2)}\right)\left\|_{\alpha}^{\left(N_{n}\right)} ;\right\| \lambda W^{(n)} \phi_{C}\left(W_{N_{n}}^{(1)}\right)+ \\
& \left.+\lambda W^{(n)} \phi_{D}\left(Z_{N_{n}}^{(1)}\right)-\lambda W^{(n)} \phi_{C}\left(W_{N_{n}}^{(2)}\right)-\lambda W^{(n)} \phi_{D}\left(Z_{N_{n}}^{(2)}\right) \|_{\alpha}^{\left(N_{n}\right)}\right\} \\
& \leq|\lambda| C_{4} \max \left\{C_{6}(A)+C_{6}(B) ; C_{6}(C)+C_{6}(D)\right\}\left\|\bar{E}_{N_{n}}^{(1)}-\bar{E}_{N_{n}}^{(2)}\right\| \\
& =\gamma\left\|\bar{E}_{N_{n}}^{(1)}-\bar{E}_{N_{n}}^{(2)}\right\|,
\end{aligned}
$$

where $\gamma<1$, for

$$
|\lambda|<\frac{1}{C_{4} \max \left\{C_{6}(A)+C_{6}(B) ; C_{6}(C)+C_{6}(D)\right\}} \equiv \lambda_{2}
$$


5. The convergence of the approximate solution of the system (1). finite.

In [4] a solution of the system (1) by the approximation method is given where $\lambda$ is

Assume that

$$
\bar{E}^{*}=\left(u^{*}, v^{*}\right) \in B\left(\bar{b} ; a_{1}\right) \subset H_{\alpha}(S) \times H_{\alpha}(S)
$$

be the unique solution of the system (1), and

$$
\bar{E}_{N_{n}}=\left(w_{N_{n}}, Z_{N_{n}}\right) \in B^{\left(N_{n}\right)}\left(\bar{b}_{N_{n}}, a_{1}\right) \subset H_{\alpha}^{\left(N_{n}\right)} \times H_{\alpha}^{\left(N_{n}\right)}
$$

be unique solution of the system of algebraic equations (13). We mean by $\bar{E}=(w, Z)$ the approximation solution of the system (1) where the functions $w(P)$ and $Z(P)$ are given by

$$
w(P)=w\left(P_{\ell}\right) \text { and } Z(P)=Z\left(P_{\ell}\right)
$$

if $P \in S\left(P_{\ell}\right), \ell=\overline{1, N_{n}}$.

We define

$$
\sup _{P \in S}\left|\bar{E}^{*}(P)-\bar{E}(P)\right| \stackrel{\text { def }}{=} \max \left\{\sup _{P \in S}\left|u^{*}(P)-w(P)\right| ; \sup _{P \in S}\left|v^{*}(P)-Z(P)\right|\right\} \text {. }
$$

If we take $0<\beta<\alpha$ we have as in [2] that

$$
\sup _{P \in S}\left|\bar{E}^{*}(P)-\bar{E}(P)\right|<C_{7} R^{\alpha-\beta}(n) \operatorname{Ln} \frac{1}{r(n)} .
$$

Theorem 5. Under a suitable choice of $|\lambda|$ we have:

(a) The system of nonlinear singular integral equation (1) and the system of nonlinear algebraic equations (11) have a unique solutions in $H_{\alpha}(S)$ and $H_{\alpha}^{\left(N_{n}\right)}$ respectively. This solution can be found by using the method of iterated approximation.

(b) The approximation solution of system (1) which is constructed by the solutions of system (11) by formula (14) converges to an exact solution with speed of order

$$
O\left(R^{\alpha-\beta}(n) \operatorname{Ln} \frac{1}{r(n)}\right), \quad 0<\beta<\alpha .
$$

\section{References}

[1] Eissa. R.P.Cubic Mechanical Method for one class of non- linear singular integral Equations "I", Bulletin of the Faculty of Engineering, Ain Shams University, No 17, 1985.

[2] Eissa R.P.Cubic Mechanical Method for one of nonlinear singular integral Equations "II", Bulletin of the Faculty of Engineering, Ain Shams University, No 17, 1985.

[3] EsKovetch E.A., The equivalence problems to the theory of two dimensional singular equation, chen. Zap. Keshenofa Univ. No 5, 1952. 
[4] EsKovetch E.A., Kolocoveskaya A.K.:The three dimensional Problems on the flowing of an ideal liquid with simple barrier. Chen. Zap. Keshen ofa Univ. No 11, 1954.

[5] Kostof U.A., Mossaef B.A.:Cubic formulae for two dimensional singularintegrals and its application. Dep. in VINITI No4281, 1981.

Eissa R.P.

Department of Mathematics, Faculty of Science, Mansoura University, Mansoura, Egypt.

Gad M.M.

Department of Mathematics, Faculty of Science, Mansoura University, Mansoura, Egypt. 